\title{
Cyclin-Dependent Kinase 5 Regulates PSD-95 Ubiquitination in Neurons
}

\author{
Michael J. Bianchetta, ${ }^{1}$ TuKiet T. Lam, ${ }^{2}$ Stephen N. Jones, ${ }^{1}$ and Maria A. Morabito ${ }^{1}$ \\ ${ }^{1}$ Department of Cell Biology, University of Massachusetts Medical School, Worcester, Massachusetts 01655 and ${ }^{2}$ W. M. Keck Foundation Biotechnology \\ Resource Laboratory, Yale University, New Haven, Connecticut 06511
}

Cyclin-dependent kinase $5(\mathrm{Cdk} 5)$ and its activator p35 have been implicated in drug addiction, neurodegenerative diseases such as Alzheimer's, learning and memory, and synapse maturation and plasticity. However, the molecular mechanisms by which Cdk 5 regulates synaptic plasticity are still unclear. PSD-95 is a major postsynaptic scaffolding protein of glutamatergic synapses that regulates synaptic strength and plasticity. PSD-95 is ubiquitinated by the ubiquitin E3 ligase Mdm2, and rapid and transient PSD-95 ubiquitination has been implicated in NMDA receptor-induced AMPA receptor endocytosis. Here we demonstrate that genetic or pharmacological reduction of Cdk5 activity increases the interaction of Mdm2 with PSD-95 and enhances PSD-95 ubiquitination without affecting PSD-95 protein levels in vivo in mice, suggesting a nonproteolytic function of ubiquitinated PSD-95 at synapses. We show that PSD-95 ubiquitination correlates with increased interaction with $\beta$-adaptin, a subunit of the clathrin adaptor protein complex AP-2. This interaction is increased by genetic reduction of Cdk5 activity or NMDA receptor stimulation and is dependent on Mdm2. Together these results support a function for Cdk5 in regulating PSD-95 ubiquitination and its interaction with AP-2 and suggest a mechanism by which PSD-95 may regulate NMDA receptor-induced AMPA receptor endocytosis.

\section{Introduction}

Cyclin-dependent kinase $5(\mathrm{Cdk} 5)$ is a proline-directed serine/ threonine kinase expressed in the CNS that, with its activator p35, is implicated in synaptic plasticity, learning and memory, drug addiction, and neurodegeneration (Angelo et al., 2006; Cheung et al., 2006; Hawasli and Bibb, 2007; Lai and Ip, 2009). Cdk5 is inactivated following NMDA receptor stimulation or depolarization (Schuman and Murase, 2003; Wei et al., 2005), and Cdk5's role in synaptic plasticity is underscored by enhanced long-term potentiation (LTP) in conditional Cdk5 knock-out mice (Hawasli et al., 2007) and a lower threshold for LTP induction and impaired long-term depression (LTD) in p35 knock-out mice (Ohshima et al., 2005; Wei et al., 2005).

PSD-95 (SAP90) is a major postsynaptic scaffolding protein of glutamatergic synapses and a substrate of Cdk5 (Morabito et al., 2004). PSD-95 has been implicated in synaptic maturation and regulation of synaptic strength and plasticity (Kim and Sheng, 2004; Funke et al., 2005; Béique et al., 2006; Elias and Nicoll, 2007). The importance of PSD-95 in synaptic plasticity is under-

Received May 12, 2011; revised June 29, 2011; accepted June 30, 2011.

Author contributions: T.T.L. and M.A.M. designed research; M.J.B. and T.T.L. performed research; S.N.J. contributed unpublished reagents/analytic tools; M.J.B., T.T.L., and M.A.M. analyzed data; M.J.B., T.T.L., S.N.J., and M.A.M. wrote the paper.

This work was supported by NIH/NIDA Grants R01DA019451 to M.A.M., R01CA077735 to S.N.J., and 1P30DA018343 to the Yale/NIDA Neuroproteomics Center. We thank Drs. L.-H. Tsai and P. Giusti for the generous contribution of p 35 knock-out mice, K. Hoover for providing assistance with Mdm2/p53 double knock-out mice and p53 knock-out mice, and the Yale/NIDA Neuroproteomics Center for their assistance. We also thank Drs. A. Rittenhouse and $Z$. Xie for comments on this manuscript.

Correspondence should be addressed to Maria A. Morabito, Department of Cell Biology, University of Massachusetts Medical School, 55 Lake Avenue North, Worcester, MA 01655. E-mail: maria.morabito@umassmed.edu.

DOI:10.1523/JNEUROSCI.2388-11.2011

Copyright $\odot 2011$ the authors $\quad 0270-6474 / 11 / 3112029-07 \$ 15.00 / 0$ scored by the inhibition of NMDA receptor (NMDAR)-induced AMPA receptor (AMPAR) internalization and the impairment of LTD following PSD-95 knockdown (Xu et al., 2008; Bhattacharyya et al., 2009). The rapid and transient ubiquitination of PSD-95 by the ubiquitin E3 ligase Mdm2 has been implicated in NMDAR-induced endocytosis of AMPARs (Colledge et al., 2003), but the mechanisms regulating this posttranslational modification of PSD-95 are still unclear.

Since Cdk5 is inactivated by NMDAR stimulation (Wei et al., 2005), we investigated whether inactivation of Cdk5 promotes PSD-95 ubiquitination. In this study we report that PSD-95 is ubiquitinated in neurons with reduced $\mathrm{Cdk} 5$ activity without affecting PSD-95 protein levels in vivo. We also show that PSD-95 ubiquitination correlates with increased interaction of PSD-95 with $\beta$-adaptin, a subunit of the clathrin adaptor protein complex AP-2, and that this interaction is increased under reduced Cdk5 activity or by NMDAR stimulation and is dependent on Mdm2. Together these results suggest a nonproteolytic signaling function for PSD-95 ubiquitination and support a novel function for $\mathrm{Cdk} 5$ in the regulation of glutamatergic synapses.

\section{Materials and Methods}

Mice. Adult p35 knock-out mice and control littermates were gifts from Dr. Li-Huei Tsai (Massachusetts Institute of Technology, Cambridge, MA). Mdm2/p53 double knock-out mice, p53 knock-out mice, and p35 knock-out mice have been described previously (Donehower et al., 1992; Jones et al., 1995; Chae et al., 1997). All mice were bred and analyzed in accordance with institutional guidelines and as approved by the Institutional Animal Care and Use Committee of the University of Massachusetts Medical School, Worcester, MA.

Acute forebrain slices. Acute forebrain slices were prepared as described previously (Zhang et al., 2008). Briefly, brains of adult wild-type mice of 
either sex were isolated, and $300 \mu \mathrm{m}$ coronal slices of forebrain (within the region corresponding approximately to -1.0 to $-3.5 \mathrm{~mm}$ from bregma, containing the hippocampus) were prepared using a Vibratome (VT1000S; Leica Microsystems) in ice-cold oxygenated artificial CSF (ACSF) containing the following (in $\mathrm{mm}$ ): $117 \mathrm{NaCl}, 1.7 \mathrm{KCl}, 1.2 \mathrm{MgCl}_{2}, 2.5 \mathrm{CaCl}_{2}, 1.2$ $\mathrm{NaH}_{2} \mathrm{PO}_{4}, 25 \mathrm{NaHCO}_{3}$, and 11.5 glucose. Slices were preincubated in ACSF for $1 \mathrm{~h}$ before treatments and all experiments were conducted at $32^{\circ} \mathrm{C}$ in $95 \% \mathrm{O}_{2}$ and $5 \% \mathrm{CO}_{2}$ incubator. Slice lysates were prepared as described previously (Zhang et al., 2008), and PSD-95 immunoprecipitated from the lysates was analyzed by Western blot and quantified as described above. Values are expressed as mean \pm SEM and were statistically compared using Student's $t$ test or a two-way ANOVA.

Western blots of brain lysates. Brain lysates were prepared according to Zhang et al., (2008). Briefly, brains (without cerebellum) were first homogenized and lysed in 1\% deoxycholate (DOC) lysis buffer $(150 \mathrm{~mm}$ $\mathrm{NaCl}, 50 \mathrm{~mm}$ Tris, $\mathrm{pH} 8.8,1 \% \mathrm{DOC})$, to which an equal volume of modified RIPA was added (150 mm NaCl, $50 \mathrm{~mm}$ Tris/HCl, pH 7.4, $1 \mathrm{~mm}$ EDTA, 1\% Triton X-100, 0.1\% SDS) (Kalia et al., 2006). All buffers were supplemented with protease and phosphatase inhibitor cocktails (Roche). Lysate protein concentrations were determined by Detergent Compatible Protein Assay (Bio-Rad). Immunoprecipitations and Western blot analysis were performed as described previously (Zhang et al., 2008). Antibodies used were mouse monoclonals Mdm2 (Millipore), PSD-95 (Antibodies Incorporated); ubiquitin (BD Transduction), FK1 (Biomol/Enzo Life Sciences), and FK2 (Biomol/Enzo Life Sciences). Western blots were visualized by enhanced chemiluminescence (GE Healthcare), and immunoreactive bands were digitally scanned and quantified with ImageJ software (NIH). Coimmunoreactivity was normalized to the level of immunoprecipitated PSD-95 and expressed as a percentage of control levels. Values are expressed as mean \pm SEM and were statistically compared using Student's $t$ test.

Mass spectrometry. PSD-95 was immunoprecipitated from acute mouse forebrain slices treated with roscovitine as described above (Acute forebrain slices). Each eluted protein sample was reduced and alkylated, digested with either LysC/Trypsin or GluC/Trypsin, and phosphoenriched with an in-house developed $\mathrm{TiO}_{2}$ enrichment strategy (Rinehart et al., 2009) to optimize for the observed $>80 \%$ sequence coverage, which encompasses 29 of the 38 lysines in mouse PSD-95. Both flowthrough and phosphoenriched fractions were injected onto a C18 RP Atlantis column attached to a nanoACQUITY UPLC system in line with a hybrid LTQ or Velos Orbitrap LC MS/MS instrument. Collected raw data from 13 experiments were processed by Distiller software (Matrix Science) and searched with the MASCOT search engine. Ubiquitinated sites were identified by the addition of a Gly-Gly moiety on lysines and were manually validated.

Dissociated hippocampal cultures and immunocytochemistry. Dissociated hippocampal cultures were prepared from E17-E18 rat brains as described previously (Zhang et al., 2008). Hippocampal neurons were plated on coverslips coated with poly-D-lysine and laminin at a density of $6600 / \mathrm{cm}^{2}$ and grown in Neurobasal medium supplemented with B27 and GlutaMax (Invitrogen) for 17-21 d in vitro. Cultures were treated for 45 min with $10 \mu \mathrm{M}$ roscovitine or DMSO as a control, fixed in $4 \%$ paraformaldehyde, $5 \%$ sucrose in PBS for $15 \mathrm{~min}$, and then permeabilized in blocking buffer (10\% BSA, $0.2 \%$ Triton X-100 in PBS) for $1 \mathrm{~h}$. Cultures were then stained using rabbit polyclonal PSD-95 antibody (Zymed) together with mouse monoclonal antibody Mdm2 (Millipore) or $\beta$-adaptin (BD Transduction Laboratories) and secondary antibodies (Alexa 488- and Alexa 594-conjugated; Invitrogen), and mounted in Vectashield (Vector Laboratories). Omission of primary antibody was used as the control for specificity of staining. Each experimental treatment and analysis was performed blind using raw images from three experiments and six coverslips. Images were captured using a Leica TCS laser-scanning confocal microscope under identical conditions $(63 \times$ objective, $1024 \times 1024$ pixel format, and identical settings). Images from each experiment $(n)$ were thresholded using identical values and analyzed by MetaMorph software. The number of costained puncta, as percentage of total PSD-95 puncta, was expressed as a percentage of the number for DMSO controls. The colocalization was expressed as mean \pm SEM and statistically compared using Student's $t$ test.
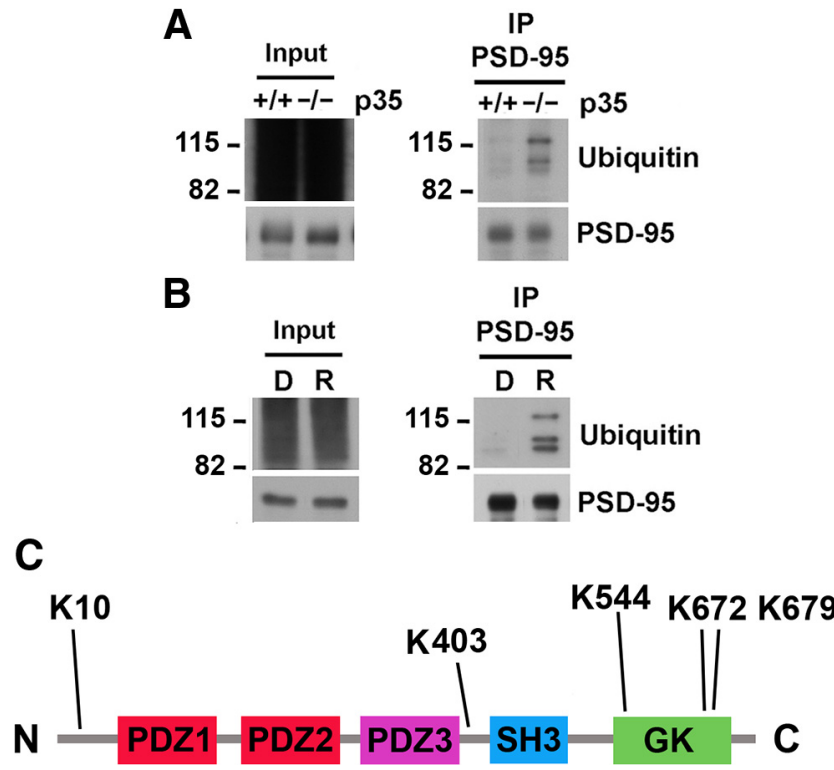

Figure 1. Reduced Cdk5 activity promotes PSD-95 ubiquitination. $\boldsymbol{A}, \mathrm{p} 35$ knock-out mice have increased PSD-95 ubiquitination. Immunoblots of PSD-95 immunoprecipitated from brain lysates of wild-type $(+/+)$ and 35 knock-out $(-/-)$ mice revealed discrete bands representing increased PSD-95 ubiquitination in p35 knock-out mice. Quantification (mean $\pm \mathrm{SEM}$ ) revealed that ubiquitination of PSD-95 was $431.3 \pm 84.4 \%$ versus $100 \pm 18.5 \%$ for wild type $(n=3 ; p<0.05)$. The ubiquitin immunoreactivity was normalized to the level of immunoprecipitated PSD-95 and expressed as a percentage of wild-type control. $\boldsymbol{B}$, Roscovitine treatment increases PSD-95 ubiquitination. Acute mouse forebrain slices were treated with the C $d \mathrm{k} 5$ inhibitor roscovitine ( $10 \mu \mathrm{m}, 45 \mathrm{~min}$ ) or DMSO as the control. Immunoblots of PSD-95 immunoprecipitated from lysates revealed increased ubiquitination of PSD-95 in roscovitine-treated (R) slices relative to control (D), with a pattern of discrete bands similar to that obtained in $\mathrm{p} 35$ knock-out mice. Quantification revealed that ubiquitination of PSD-95 was $274.6 \pm 71.6 \%$ with roscovitine treatment versus $100 \pm 54.5 \%$ in untreated control ( $n=4 ; p<0.01)$. Ubiquitin immunoreactivity was normalized to the level of immunoprecipitated PSD-95 and expressed as a percentage of DMSO control. C, PSD-95 is ubiquitinated on multiple lysines. PSD-95 immunoprecipitated from acute mouse forebrain slices treated with the Cdk5 inhibitor roscovitine (10 $\mu \mathrm{M}, 45 \mathrm{~min}$ ) was subjected to LCMS/MS analysis. Peptides obtained covered $>80 \%$ of the PSD-95 primary amino acid sequence (DLG4, mouse; Swiss-Prot database). Aschematic view of PSD-95 domain structure with ubiquitinated lysines (K) is shown. Lysine residues ubiquitinated are $\mathrm{K} 10$ in the N terminus, K 403 in the linker between the PDZ3 and SH3 domains, and K 544 , $K 672$, and $K 679$ in the GK domain.

\section{Results}

\section{Decreased Cdk5 activity increases PSD-95 ubiquitination}

To assess whether Cdk5 regulates PSD-95 ubiquitination, we analyzed the levels of PSD-95 ubiquitination in p35 knock-out mice, which have reduced Cdk5 activity by $78 \%$ (Hallows et al., 2006). Immunoblotting of PSD-95 immunoprecipitated from brain revealed a more than fourfold increase in PSD-95 ubiquitination in p35 knock-out mice compared to wild-type littermates $(431.3 \pm 84.4 \%$ vs $100 \pm 18.5 \%$ for wild type; $n=3 ; p<0.05$; Fig. $1 A)$, with a pattern of discrete bands closely resembling those observed by Colledge et al. (2003). We also determined the levels of PSD-95 ubiquitination in acute mouse forebrain slices in which Cdk5 was inhibited pharmacologically by treatment with the Cdk5 inhibitor roscovitine (Fig. $1 B$ ). Consistent with the increase in PSD-95 ubiquitination observed in p35 knock-out mice, pharmacological inhibition of Cdk 5 by roscovitine resulted in a more than twofold increase in PSD-95 ubiquitination (274.6 $\pm 71.6 \%$ vs $100 \pm 54.5 \%$ for untreated control; $n=4 ; p<$ $0.01)$. Together, these data indicate that decreased Cdk5 activity results in increased PSD-95 ubiquitination.

PSD-95 contains multiple protein-protein interaction motifs including an N-terminal domain, three PDZ domains, an SH3 
(Src homology 3) domain, and a GK (guanylate kinase) domain (Kim and Sheng, 2004; Montgomery et al., 2004). To identify the ubiquitinated lysines on PSD-95, we performed high-resolution tandem mass spectrometry analysis on PSD-95 immunoprecipitated from acute mouse forebrain slices treated with roscovitine. High molecular weight (MW) and long peptides generally pose difficulty in collision-induced dissociation-type fragmentation by most tandem mass spectrometers. Based on theoretically predicted digestion using various enzymes, we found that by including either GluC (Staphylococcus aureus protease V8) or LysC (endoproteinase from Lysobacter enzymogenes) with a trypsin digestion of PSD-95, we eliminated all the high MW peptides (peptides with $\mathrm{MW}>\sim 3500$ ) generated from just using trypsin. The combination of $\mathrm{GluC}$ or LysC with trypsin digestion resulted in $>80 \%$ primary protein sequence coverage of PSD-95 (DLG4, mouse; Swiss-Prot database), overlapping 29 of the 38 lysines, with Lys 152, Lys 157, Lys 162, Lys 165, Lys 211, Lys 355, Lys 503, Lys 505, and Lys 624, not represented. The presence of a GlyGly moiety on a lysine residue corresponds to the $\mathrm{C}$ terminus of the ubiquitin tag, which is present when trypsin (or a combination of GluC/trypsin enzymes) is used for enzymatic digestion before LC MS/MS analysis, and is indicative of ubiquitination. We identified five lysine residues of PSD-95 that were ubiquitinated after roscovitine treatment (Fig. 1C): Lys 10 in the $\mathrm{N}$ terminus, Lys 403 in the linker between PDZ3 and SH3 domains, and Lys 544, Lys 672, and Lys 679 in the GK domain. These results indicate that, under decreased Cdk5 activity, the $\mathrm{N}$ terminus as well as the $\mathrm{PDZ} 3 / \mathrm{SH} 3$ linker region and the GK domain are ubiquitinated.

$\mathrm{Mdm} 2$ has been identified as the ubiquitin ligase for PSD-95 (Colledge et al., 2003). Since the ubiquitination of PSD-95 is dependent on Mdm2, the increase in PSD-95 ubiquitination that we observed under reduced Cdk5 activity could reflect an increased interaction of PSD-95 with Mdm2. To investigate whether Cdk5 activity inversely correlates with the level of interaction of PSD-95 with Mdm2, we analyzed PSD-95 immunoprecipitated from brain lysates of p35 knock-out mice and wild-type littermates by immunoblotting with an Mdm2 antibody (Fig. $2 A)$. Consistent with the increase in PSD-95 ubiquitination, the interaction of Mdm2 with PSD-95 increased more than threefold in $\mathrm{p} 35 \mathrm{knock}$-out mice compared to wild type ( $360.5 \pm 65.6 \%$ vs $100 \pm 20.6 \%$ for wild type; $n=3 ; p<0.05$ ). To further assess whether Cdk5 regulates the level of PSD-95 association with $\mathrm{Mdm} 2$, we analyzed roscovitine-treated cultured hippocampal neurons by immunocytochemistry (Fig. $2 \mathrm{~B}$ ). We found that roscovitine treatment increased the colocalization of Mdm2 with PSD-95 puncta compared to DMSO control sister cultures (149 $\pm 27.4 \%$ vs $100 \pm 24.8 \%$ for control; $n=4 ; p<0.05$ ). Together these data indicate that decreased Cdk5 activity increases colocalization and interaction of Mdm2 with PSD-95, thus promoting PSD-95 ubiquitination by $\mathrm{Mdm} 2$.

\section{Decreased Cdk5 activity increases PSD-95 ubiquitination without a decrease in PSD-95 protein levels}

Ubiquitination may occur by the addition of a single ubiquitin moiety to one or multiple lysine residues (monoubiquitination) or by addition of polymeric ubiquitin chains (polyubiquitination) (DiAntonio and Hicke, 2004; d'Azzo et al., 2005). While $\mathrm{Mdm} 2$ can catalyze both monoubiquitination and polyubiquitination (Li et al., 2003), the discrete ubiquitinated PSD-95 species that we observe and that were originally identified by Colledge et al. (2003) suggest PSD-95 monoubiquitination on multiple sites. The FK1 and FK2 monoclonal antibodies are used to differentiate
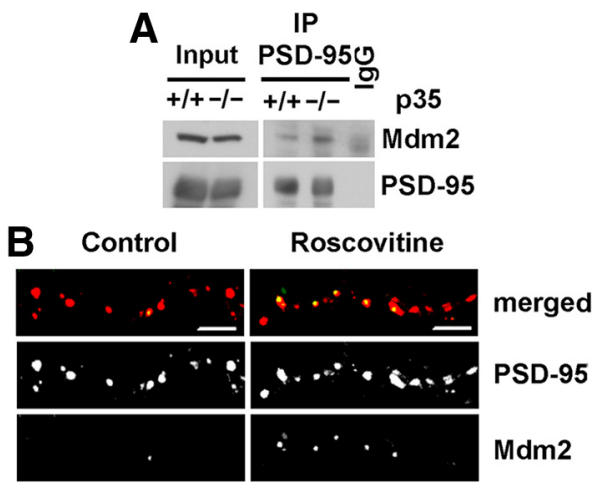

Figure 2. Reduced Cdk5 activity increases interaction and colocalization of Mdm2 with PSD95. $\boldsymbol{A}, \mathrm{p} 35$ knock-out mice have increased interaction of Mdm2 with PSD-95. Immunoblots of PSD-95 immunoprecipitated from brain lysates of wild-type $(+/+)$ and p35 knock-out $(-/-)$ mice revealed increased interaction of PSD-95 with Mdm2 in p35 knock-out mice. Quantification (mean \pm SEM) revealed that coimmunoprecipitation of Mdm 2 with PSD-95 was $360.5 \pm 65.6 \%$ versus $100 \pm 20.6 \%$ for wild type $(n=3 ; p<0.05)$. Mdm 2 immunoreactivity was normalized to the level of immunoprecipitated PSD-95 and expressed as a percentage of wild-type control. IgG, Control immunoprecipitation with mouse lgG. $\boldsymbol{B}$, Roscovitine treatment increases the colocalization of Mdm2 with PSD-95. Cultured hippocampal neurons were treated with the C $\mathrm{dk} 5$ inhibitor roscovitine ( $10 \mu \mathrm{M}, 45 \mathrm{~min}$ ) or DMSO as a control and immunostained for Mdm2 and PSD-95. Representative confocal images from each condition are shown (Mdm2, green; PSD-95, red). Scale bars: $10 \mu \mathrm{m}$. Quantification revealed that in roscovitine-treated cultures (R), the percentage of PSD-95 puncta positive for Mdm2 was $149 \pm 27.4 \%$ versus $100 \pm 24.8 \%$ the DMSO control (D) ( $n=4 ; p<0.05)$. The number of costained puncta, as a percentage of total PSD-95 puncta, is expressed as a percentage of the number for DMSO controls.

between monoubiquitination and polyubiquitination, since FK2 detects both monoubiquitinated and polyubiquitinated proteins, while FK1 recognizes only polyubiquitinated proteins (Fujimuro et al., 1994). Therefore, to assess whether the ubiquitination of PSD-95 reflects monoubiquitination or polyubiquitination, we tested PSD-95 from p35 knock-out mice for immunoblotting with FK1 and FK2 antibodies. Immunoblotting of PSD-95 immunoprecipitated from p35 knock-out brain revealed that PSD-95 ubiquitination is detected by FK2, not FK1. Polyubiquitinated species observed in the input lanes serve as a positive control for FK1 immunoreactivity (Fig. 3A). Similar results were obtained with PSD-95 immunoprecipitated from roscovitinetreated acute forebrain slices (Fig. 3B). The immunoreactivity of PSD-95 to FK2 but not to FK1 suggests monoubiquitination of PSD-95 on multiple lysines and is consistent with the LC MS/MS results.

While polyubiquitination has been mainly associated with targeting of proteins for degradation by the $26 \mathrm{~S}$ proteasome, monoubiquitination has been implicated in nonproteasomal signaling functions (Hicke and Dunn, 2003; DiAntonio and Hicke, 2004; d'Azzo et al., 2005; Chen and Sun 2009). To assess the relationship between PSD-95 ubiquitination and degradation, we analyzed the levels of PSD-95 in brain lysates of p35 knock-out and wild-type mice (Fig. 3C) and in roscovitine-treated and control acute forebrain slices (Fig. 3D). We observed no significant change in PSD-95 protein levels (normalized to actin) in p35 knock-out mice compared to wild-type littermates (120.7 \pm $30.5 \%$ vs $100 \pm 15.7 \%$ for wild type; $n=4 ; p>0.5)$ or in roscovitine-treated slices compared to control (103.7 $\pm 18.7 \%$ vs $100 \pm 15.8 \%$ for control; $n=3 ; p>0.5)$. Thus, these results indicate that PSD-95 ubiquitination does not directly correlate with decreased PSD-95 protein levels, suggesting a nonproteolytic function for ubiquitinated PSD-95 at the synapse. 
A

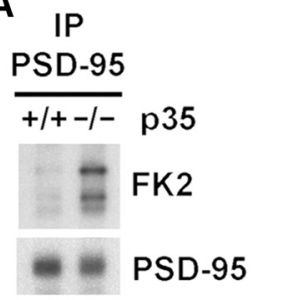

B

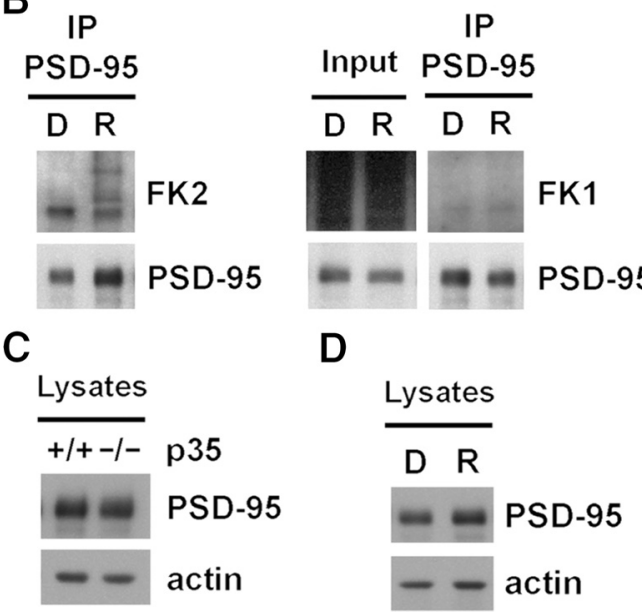

Figure 3. Ubiquitination of PSD-95 does not alter PSD-95 protein levels. A, PSD-95 is monoubiquitinated on multiple lysines in p35 knock-out mice. PSD-95 immunoprecipitated from brain lysates of wild-type $(+/+)$ and p35 knock-out $(-/-)$ mice immunoblotted with the FK2 antibody, which recognizes polyubiquitinated and monoubiquitinated proteins. FK2 immunoblot analysis revealed increased PSD-95 ubiquitination in p35 knock-out mice, with the same pattern of discrete bands observed with the ubiquitin antibody. Immunoblot with the FK1 antibody, which recognizes only polyubiquitinated proteins, did not reveal immunoreactive bands (right). Immunoblotting of polyubiquitinated proteins in the input serves as a positive control for FK1. B, Roscovitine treatment increases PSD-95 monoubiquitination on multiple lysines. Acute mouse forebrain slices were treated with the $C \mathrm{dk} 5$ inhibitor roscovitine ( $R ; 10 \mu \mathrm{m}$, $45 \mathrm{~min}$ ) or DMSO (D) as a control. Immunoblots of PSD-95 immunoprecipitated from lysates of the treated slices revealed FK2 immunoreactivity with a pattern of discrete bands similar to that obtained in p35 knock-out mice. FK1 immunoblots did not reveal immunoreactive bands from immunoprecipitated PSD-95 as for the p35 knock-out mice (right). Immunoblotting of polyubiquitinated proteins in the input serves as a positive control for FK1. C, PSD-95 ubiquitination does not alter PSD-95 protein levels. Brain lysates from wild-type $(+/+)$ and p35 knock-out $(-/-)$ mice were immunoblotted for PSD-95 and actin. Quantification (mean \pm SEM) revealed that PSD-95 protein levels in the 35 knock-out mice were $120.7 \pm 30.5 \%$ versus $100 \pm$ $15.7 \%$ for wild type $(n=4 ; p>0.5)$. PSD-95 immunoreactivity was normalized to actin and expressed as a percentage of wild-type control mice. $\boldsymbol{D}$, Roscovitine treatment does not affect the protein levels of PSD-95. Brain lysates from acute mouse forebrain slices treated with the Cdk5 inhibitor roscovitine (10 $\mu \mathrm{M}, 45 \mathrm{~min}$ ) or DMSO as control were immunoblotted for PSD-95 and actin. Quantification revealed that PSD-95 protein levels in the roscovitine samples were $103.7 \pm 18.7 \%$ versus $100 \pm 15.8 \%$ for DMSO controls $(n=3 ; p>0.5)$. PSD- 95 immunoreactivity was normalized to actin and expressed as a percentage of DMSO control levels.

PSD-95 ubiquitination promotes the interaction with the clathrin endocytic adaptor complex AP-2

AMPAR endocytosis is mediated by the clathrin endocytic adaptor complex AP-2 (Carroll et al., 1999; Man et al., 2000). While the function of PSD-95 in AMPAR endocytosis is not clear, it has been shown that PSD-95 interacts with the AP-2 complex (Fernandez et al., 2009), and the AP-2 binding motif (YXXФ) within the GK domain of PSD-95 ( $\left.\mathrm{Y}_{701} \mathrm{HKV}\right)$ is sufficient to mediate clathrin-dependent endocytosis of the transmembrane protein Tac (Craven and Bredt, 2000). Therefore, we hypothesize that PSD-95 ubiquitination functions by regulating the interaction of PSD-95 with the AP-2 complex.
Since p35 knock-out mice have increased PSD-95 ubiquitination, we used these mutant mice to assess whether PSD-95 ubiquitination correlates with changes in PSD-95 interaction with AP-2 in vivo. We analyzed the interaction of PSD-95 with AP-2 in brain lysates of p35 knock-out and wild-type littermates by immunoblotting immunoprecipitated PSD-95 for the AP-2 subunit $\beta$-adaptin (Fig. $4 A$ ). The interaction of PSD-95 with $\beta$-adaptin was significantly increased in p35 knock-out mice (265 $\pm 55.8 \%)$ versus wild type $(100 \pm 16.4 \% ; n=4 ; p<0.05)$. We also analyzed whether $\beta$-adaptin colocalizes with PSD-95 puncta in cultured hippocampal neurons treated with roscovitine (Fig. $4 B$ ). Immunocytochemistry analysis of these neurons revealed that roscovitine treatment promotes colocalization of $\beta$-adaptin with PSD-95 puncta compared to DMSO-treated control sister neurons $(192.5 \pm 19 \%$ vs $100 \pm 15.6 \%$ for control; $n=5 ; p<0.01)$. Thus, these data indicate that decreased Cdk5 activity, which promotes PSD-95 ubiquitination, also promotes the interaction of PSD-95 with $\beta$-adaptin. To further assess whether PSD-95 ubiquitination regulates the interaction with AP-2, we analyzed PSD-95 immunoprecipitated from brain lysates of Mdm2/p53 double knock-out mice, since Mdm2 knock-out mice die early in development (Jones et al., 1995), and control p53 knock-out mice (Fig. 4C). Immunoblot analysis of $\mathrm{Mdm} 2 / \mathrm{p} 53$ double knock-out brain lysates revealed decreased interaction of PSD-95 with $\beta$-adaptin compared to control p53 knock-out mice (63.7 \pm $8.8 \%$ vs $100 \pm 10.8 \%$ for control; $n=5 ; p<0.05)$. Together, these results indicate a correlation between the genetic deletion of Mdm2 and the decrease in PSD-95 interaction with $\beta$-adaptin and, conversely, between decreased Cdk 5 activity and increased PSD-95 interaction with $\beta$-adaptin. Therefore, these results support a correlation between PSD-95 ubiquitination and interaction with $\beta$-adaptin, suggesting a function for ubiquitinated PSD-95 in the regulation of its interaction with the AP-2/clathrin endocytic complex.

PSD-95 ubiquitination is also induced by NMDA treatment (Colledge et al., 2003); thus, we investigated whether NMDA treatment regulates the interaction of PSD-95 with $\beta$-adaptin. Acute forebrain slices from adult wild-type mice were treated with NMDA (100 $\mu \mathrm{M}, 3 \mathrm{~min})$, a treatment that induces AMPAR endocytosis (Bhattacharyya et al., 2009), and analyzed at 5 and 10 min after NMDA washout. Immunoblot analysis of PSD-95 immunoprecipitated from the lysates revealed an increase in both PSD-95 ubiquitination and interaction with $\beta$-adaptin $10 \mathrm{~min}$ after NMDA washout (Fig. 4D). To further assess the correlation between PSD-95 ubiquitination and interaction with $\beta$-adaptin, we analyzed acute brain slices of adult Mdm2/p53 double knockout mice and control p53 knock-out mice 5 and 10 min after NMDA (100 $\mu \mathrm{M}, 3 \mathrm{~min}$ ) washout (Fig. $4 E$ ). Immunoblot analysis of PSD-95 immunoprecipitated from lysates of control p53 knock-out mice revealed an increase in PSD-95 interaction with $\beta$-adaptin at $5 \min (209.0 \pm 72.6 \% ; n=3)$ and $10 \min (549.8 \pm$ $168 \% ; n=3$ ) after washout compared to the unstimulated control condition $(100 \pm 14.9 \% ; n=3)$. In contrast, the interaction of PSD-95 with $\beta$-adaptin was consistently lower in Mdm2/p53 double knock-out slices in unstimulated conditions (50.5 \pm $13.8 \% ; n=3)$ at $5 \min (45.6 \pm 1.8 \% ; n=3)$ and $10 \min (99.8 \pm$ $43.7 \% ; n=3$ ) after NMDA washout compared to unstimulated p53 knock-out control conditions. All the values obtained for the PSD-95/ $\beta$-adaptin interaction in p53 and $\mathrm{Mdm} 2 / \mathrm{p} 53$ double knock-outs were statistically significant $(p<0.005$, ANOVA). These results indicate that the interaction of PSD-95 with $\beta$-adaptin is regulated by NMDARs and depends on Mdm2 expression. Thus, together these data support a direct correlation 
A

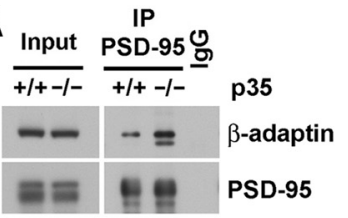

C

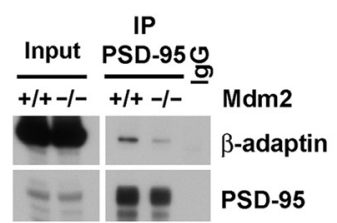

$\mathbf{E}$

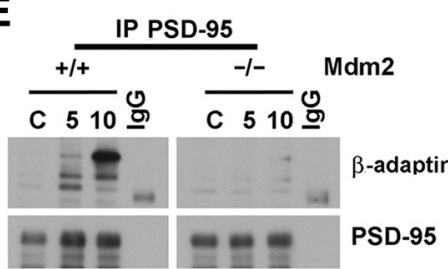

Figure 4. PSD-95 ubiquitination correlates with PSD-95 interaction with AP-2. A, p35 knock-out mice have increased PSD-95 interaction with $\beta$-adaptin, a subunit of the clathrin endocytic adaptor complex AP-2. Immunoblots of PSD-95 immunoprecipitated from brain lysates of p35 knock-out $(-/-)$ and wild-type $(+/+)$ mice revealed increased interaction of PSD-95 with $\beta$-adaptin in $\mathrm{p} 35$ knock-outs. Quantification (mean \pm SEM) revealed that the coimmunoprecipitation of $\beta$-adaptin with PSD-95 was $265 \pm 55.8 \%$ of $100 \pm 16.4 \%$ for wild type $(n=4 ; p<0.05)$. $\beta$-Adaptin immunoreactivity was normalized to the level of immunoprecipitated PSD-95 and expressed as a percentage of wild-type control. lgG, Control immunoprecipitation with mouse $\lg$ G. $B$, Pharmacological inhibition of C $d k 5$ increases the colocalization of PSD-95 with $\beta$-adaptin. Cultured hippocampal neurons treated with the specific $\mathrm{Cdk} 5$ inhibitor roscovitine ( $10 \mu \mathrm{m}, 45 \mathrm{~min}$ ) or DMSO as a control were immunostained with $\beta$-adaptin and PSD-95 antibodies. Representative confocal images from each condition are shown ( $\beta$-adaptin, green; PSD-95, red). Scale bars: 10 $\mu \mathrm{m}$. Quantification revealed that in roscovitine-treated cultures $(\mathrm{R})$ recruiting the clathrin/AP-2 endocytic complex, the percentage of PSD-95 puncta positive for $\beta$-adaptin was $192.5 \pm 19 \%$ of $100 \pm 15.6 \%$ for the DMSO control $(D ; n=5 ; p<0.01)$. The number of costained puncta, as a percentage of total PSD-95 puncta, is expressed as a percentage of the number for DMSO controls. C, Mdm 2 null mice have decreased interaction of PSD-95 with $\beta$-adaptin. Immunoblots of PSD-95 immunoprecipitated from brain lysates of p53/Mdm2 double knock-out ( - / - ) and p53 knock-out control mice $(+/+)$ revealed decreased interaction of PSD-95 with $\beta$-adaptin in $\mathrm{p53} / \mathrm{Mdm} 2$ double knock-out mice. Quantification revealed that coimmunoprecipitation of $\beta$-adaptin with PSD-95 was $63.7 \pm 8.8 \%$ of $100 \pm 10.8 \%$ for the $\mathrm{p} 53$ knock-out control $(n=5 ; p<0.05)$. $\beta$-Adaptin immunoreactivity was normalized to the level of immunoprecipitated PSD-95 and expressed as a percentage of p53 knock-out control levels. D, NMDA receptors regulate PSD-95 ubiquitination and interaction with $\beta$-adaptin. Acute forebrain slices from wild-type mice were stimulated with NMDA (100 $\mu \mathrm{m}, 3 \mathrm{~min}$ ) and, after NMDA washout, incubated for an additional 5 or 10 min. Immunoblots of PSD-95 immunoprecipitated from lysates revealed increased coimmunoprecipitation of $\beta$-adaptin with PSD-95 10 min after NMDA washout (10) compared to the unstimulated control (C). FK2 immunoblots of PSD-95 revealed increased PSD-95 ubiquitination 10 min after NMDA washout (10) compared to the unstimulated control (C). $E, M d m 2$ null mice have reduced NMDA-dependent interaction of PSD-95 with $\beta$-adaptin. Acute forebrain slices from control p53 knock-out $(+/+)$ and Mdm2/p53 double knock-out mice $(-/-)$ were stimulated with NMDA (100 $\mu \mathrm{m}, 3 \mathrm{~min})$ and, after NMDA washout, incubated for an additional 5 or $10 \mathrm{~min}$ Quantification of immunoblots of PSD-95 immunoprecipitated from $\mathrm{p} 53$ knock-out lysates revealed increased coimmunoprecipitation of $\beta$-adaptin with PSD-95 5 min after the end of NMDA treatment $(209.0 \pm 72.6 \% ; n=3)$, and a further increase at $10 \mathrm{~min}$ $(549.8 \pm 167.7 \% ; n=3)$ compared to the unstimulated control $(100 \pm 14.9 \% ; n=3)$. In contrast, compared to the unstimulated p53 knock-out control, the levels of coimmunoprecipitation of $\beta$-adaptin with PSD-95 were lower in Mdm2/p53 double knock-out mice at all times points in unstimulated slices $(50.5 \pm 13.8 \% ; n=3)$ as well as at 5 and 10 min after the end of NMDA treatment ( $45.6 \pm 1.8 \%$ and $99.8 \pm 43.7 \%$, respectively; $n=3 ; p<0.005$, ANOVA). $\beta$-Adaptin immunoreactivity was normalized to the level of immunoprecipitated PSD-95 and expressed as a percentage of p53 knock-out unstimulated controls.

between PSD-95 ubiquitination and interaction with AP-2, suggesting that PSD-95 ubiquitination may function in NMDARdependent AMPAR endocytosis by regulating the interaction with the clathrin endocytic adaptor complex AP-2.

\section{Discussion}

Cdk 5 has been implicated in synaptic plasticity, but the molecular mechanisms are still not clear. PSD-95 ubiquitination by Mdm2 is stimulated by NMDAR activity and has been implicated in the regulation of NMDAR-induced internalization of AMPARs during synaptic plasticity. Here we show that PSD-95 ubiquitination is increased by genetic or pharmacological reduction of Cdk5 activity reflects ubiquitination on multiple lysines and does not correlate with decreased PSD-95 protein levels in vivo. We also show that PSD-95 ubiquitination correlates with increased interaction with the AP-2 complex both in p35 knock-out mice, which have reduced Cdk5 activity, and by direct stimulation of acute forebrain slices with NMDA. Together these results suggest a molecular mechanism by which Cdk5 impacts synaptic plasticity by regulating PSD-95 ubiquitination and its interaction with AP-2.

We provide evidence that reduced Cdk5 activity promotes the colocalization and interaction of Mdm2 with PSD-95 and the ubiquitination of PSD-95 in neurons. $\mathrm{Mdm} 2$ activity is regulated by numerous signaling pathways and is affected by changes in its stability and intracellular distribution (Mukhopadhyay and Riezman, 2007; Wade et al., 2010). Thus, reduced Cdk5 activity may promote PSD-95 ubiquitination by regulating the intracellular distribution of Mdm2 within neurons, possibly by increasing Mdm2 levels at the PSD. Mdm2 can catalyze both monoubiquitination and polyubiquitination of the tumor suppressor p53 (Li et al., 2003) and the pattern of discrete bands of ubiquitinated PSD-95 observed by Colledge et al., (2003), and in our experiments, suggests monoubiquitination on multiple lysines. Our study, using the differential immunoreactivity to the FK1 and FK2 antibodies, is consistent with PSD-95 monoubiquitination on multiple lysines. We have also identified, by high-resolution mass spectrometry, five ubiquitinated residues in PSD-95 from roscovitine-treated samples, although some regions of PSD-95 were not covered by our analysis, possibly due to ions not being ionized in the positive mode in the mass spectrometer, therefore making the peptides more observable in the negative mode (Toll et al., 2005). The identification of five ubiquitinated lysine residues in PSD-95 suggests that the three distinct bands of ubiquitinated PSD-95 observed in our experiments and by Colledge et al., (2003) may represent different combinations of ubiquitinated residues, with the high molecular weight band being consistent with three ubiquitinated residues. Interestingly, one of the lysines (Lys 10) is within the N-terminal domain of PSD-95, which has been implicated in homotypic interactions (Hsueh et al., 1997; Morabito et al., 2004; Xu et al., 2008). Lysine 10 is also adjacent to the PEST [proline $(\mathrm{P})$, glutamic acid $(\mathrm{E})$, serine $(\mathrm{S})$, and threonine $(\mathrm{T})$ rich] motif of PSD-95, which is required for NMDAR-induced AMPAR endocytosis (Colledge et al., 2003). Additional ubiquitinated lysines are in the linker between the PDZ3 and SH3 domains (Lys 403) and within the GK domain (Lys 544, Lys 672, and Lys 679). The 
SH3 and GK domains are linked by a pair of antiparallel strands and form an integrated structural unit (McGee et al., 2001; Tavares et al., 2001). Lys 544, Lys 672, and Lys 679 are within $\alpha$ helix structures of the GK domain, suggesting that ubiquitination of these residues may change the conformation of the SH3/GK domain through the sterical presence of the ubiquitin moiety, making PSD-95 more accessible for protein interactions. In addition, the SH3 and GK domains are important for the localization of PSD-95 at the PSD, and hence for the effect of PSD-95 on AMPAR EPSCs (Xu et al., 2008). Thus, addition of ubiquitin moieties to these domains may regulate PSD-95 protein interactions, the organization of signaling molecules and cytoskeletal elements, and the strength of synaptic transmission.

In our study, PSD-95 ubiquitination does not correlate with its degradation both in p35 knock-out brain in vivo and in roscovitine-treated neurons, and is consistent with PSD-95 monoubiquitination. Previous studies have associated PSD-95 ubiquitination with its proteasomal degradation by showing that deletion of the PEST motif in PSD-95 or treatment with the proteasome inhibitor MG132 $\left(\mathrm{C}_{26} \mathrm{H}_{41} \mathrm{~N}_{3} \mathrm{O}_{5}\right)$ protects PSD-95 from degradation (Colledge et al., 2003). Interestingly, the proteases calpain and caspase can catalyze PSD-95 degradation (Lu et al., 2000; Vinade et al., 2001; Gascón et al., 2008; Liu et al., 2010), and MG132 is also an inhibitor of calpains (Lee and Goldberg, 1998; Elliott et al., 2003). Furthermore, Lys 10 in PSD-95, which is ubiquitinated under reduced $\operatorname{Cdk} 5$ activity, is adjacent to the PEST motif, which has been implicated in proteolysis by caspases (Belizario et al., 2008) and calpains (Rechsteiner and Rogers, 1996; Shumway et al., 1999).

Our studies suggest a novel function for ubiquitinated PSD-95 at the synapse in the regulation of PSD-95 interaction with the clathrin endocytic adaptor complex AP-2. We observe a correlation between reduction of Cdk5 activity, increased PSD-95 ubiquitination, and increased PSD-95 interaction with $\beta$-adaptin, a component of AP-2/clathrin adaptor protein complex. We also observe the NMDAR-dependent increase in PSD-95 ubiquitination, first described by Colledge et al., (2003), accompanied by the increase in PSD-95 interaction with $\beta$-adaptin. The correlation between PSD-95 ubiquitination and increased interaction with AP-2 is further supported by our observation that, whereas in p53 knock-out mice NMDAR activity induces an increase in PSD-95 interaction with $\beta$-adaptin, this increase is greatly reduced in mice with a genetic deletion of $\mathrm{Mdm} 2$. Our results are consistent with previous studies indicating an interaction between PSD-95 and AP-2 (Fernandez et al., 2009) and a function for the motif within the GK domain of PSD-95 in mediating the clathrin-dependent endocytosis of the transmembrane protein Tac (Craven and Bredt, 2000). Together these observations suggest that ubiquitinated PSD-95 functions in recruiting the clathrin/AP-2 endocytic complex and possibly regulating the interaction with other postsynaptic proteins. Our data demonstrate that Cdk5 regulates the ubiquitination of PSD-95 and its interaction with AP-2. Given that ubiquitination of PSD-95 has been implicated in NMDAR-induced endocytosis of AMPARs, the recruitment of the clathrin/AP-2 endocytic complex by PSD-95 could provide a mechanism by which Cdk 5 could modulate AMPAR internalization during synaptic plasticity.

\section{References}

Angelo M, Plattner F, Giese KP (2006) Cyclin-dependent kinase 5 in synaptic plasticity, learning and memory. J Neurochem 99:353-370.

Béique JC, Lin DT, Kang MG, Aizawa H, Takamiya K, Huganir RL (2006) Synapse-specific regulation of AMPA receptor function by PSD-95. Proc Natl Acad Sci U S A 103:19535-19540.

Belizario JE, Alves J, Garay-Malpartida M, Occhiucci JM (2008) Coupling caspase cleavage and proteasomal degradation of proteins carrying PEST motif. Curr Protein Pept Sci 9:210-220.

Bhattacharyya S, Biou V, Xu W, Schlüter O, Malenka RC (2009) A critical role for PSD-95/AKAP interactions in endocytosis of synaptic AMPA receptors. Nat Neurosci 12:172-181.

Carroll RC, Beattie EC, Xia H, Lüscher C, Altschuler Y, Nicoll RA, Malenka RC, von Zastrow M (1999) Dynamin-dependent endocytosis of ionotropic glutamate receptors. Proc Natl Acad Sci U S A 96:14112-14117.

Chae T, Kwon YT, Bronson R, Dikkes P, Li E, Tsai LH (1997) Mice lacking p35, a neuronal specific activator of Cdk5, display cortical lamination defects, seizures, and adult lethality. Neuron 18:29-42.

Chen ZJ, Sun LJ (2009) Nonproteolytic functions of ubiquitin in cell signaling. Mol Cell 33:275-286.

Cheung ZH, Fu AK, Ip NY (2006) Synaptic roles of Cdk5: implications in higher cognitive functions and neurodegenerative diseases. Neuron 50:13-18.

Colledge M, Snyder EM, Crozier RA, Soderling JA, Jin Y, Langeberg LK, Lu H, Bear MF, Scott JD (2003) Ubiquitination regulates PSD-95 degradation and AMPA receptor surface expression. Neuron 40:595-607.

Craven SE, Bredt DS (2000) Synaptic targeting of the postsynaptic density protein PSD-95 mediated by a tyrosine-based trafficking signal. J Biol Chem 275:20045-20051.

d'Azzo A, Bongiovanni A, Nastasi T (2005) E3 ubiquitin ligases as regulators of membrane protein trafficking and degradation. Traffic 6:429-441.

DiAntonio A, Hicke L (2004) Ubiquitin-dependent regulation of the synapse. Annu Rev Neurosci 27:223-246.

Donehower LA, Harvey M, Slagle BL, McArthur MJ, Montgomery CA Jr, Butel JS, Bradley A (1992) Mice deficient for p53 are developmentally normal but susceptible to spontaneous tumours. Nature 356:215-221.

Elias GM, Nicoll RA (2007) Synaptic trafficking of glutamate receptors by MAGUK scaffolding proteins. Trends Cell Biol 17:343-352.

Elliott PJ, Zollner TM, Boehncke W.H., (2003) Proteasome inhibition: a new anti-inflammatory strategy, J Mol Med 81:235-245.

Fernandez E, Collins MO, Uren RT, Kopanitsa MV, Komiyama NH, Croning MDR, Zografos L, Armstrong JD, Choudhary JS, Grant SG (2009) Targeted tandem affinity purification of PSD-95 recovers core postsynaptic complexes and schizophrenia susceptibility proteins. Mol Sys Biol 5:269.

Fujimuro M, Sawada H, Yokosawa H (1994) Production and characterization of monoclonal antibodies specific to multi-ubiquitin chains of polyubiquitinated proteins. FEBS Lett 349:173-180.

Funke L, Dakoji S, Bredt DS (2005) Membrane-associated guanylate kinases regulate adhesion and plasticity at cell junctions. Annu Rev Biochem 74:219-245.

Gascón S, Sobrado M, Roda JM, Rodríguez-Peña A, Díaz-Guerra M (2008) Excitotoxicity and focal cerebral ischemia induce truncation of the NR2A and NR2B subunits of the NMDA receptor and cleavage of the scaffolding protein PSD-95. Mol Psychiatry 13:99-114.

Hallows JL, Iosif RE, Biasell RD, Vincent I (2006) p35/p25 is not essential for tau and cytoskeletal pathology or neuronal loss in Niemann-Pick type C disease. J Neurosci 26:2738-2744.

Hawasli AH, Bibb JA (2007) Alternative roles for Cdk5 in learning and synaptic plasticity. Biotechnol J 2:941-948.

Hawasli AH, Benavides DR, Nguyen C, Kansy JW, Hayashi K, Chambon P, Greengard P, Powell CM, Cooper DC, Bibb JA (2007) Cyclindependent kinase 5 governs learning and synaptic plasticity via control of NMDAR degradation. Nat. Neurosci 10:880-886.

Hicke L, Dunn R (2003) Regulation of membrane protein transport by ubiquitin and ubiquitin-binding proteins. Annu Rev Cell Dev Biol 19:141-172.

Hsueh YP, Kim E, Sheng M (1997) Disulfide-linked head-to-head multimerization in the mechanism of ion channel clustering by PSD-95. Neuron 18:803-814.

Jones SN, Roe AE, Donehower LA, Bradley A (1995) Rescue of embryonic lethality in Mdm2-deficient mice by absence of p53. Nature 378:206-208.

Kalia LV, Pitcher GM, Pelkey KA, Salter MW (2006) PSD-95 is a negative regulator of the tyrosine kinase Src in the NMDA receptor complex. EMBO J 25:4971-4982.

Kim E, Sheng M (2004) PDZ domain proteins of synapses. Nat Rev Neurosci 5:771-781.

Lai KO, Ip NY (2009) Recent advances in understanding the roles of Cdk5 in synaptic plasticity. Biochim Biophys Acta 1792:741-745. 
Lee DH, Goldberg AL (1998) Proteasome inhibitors: valuable new tools for cell biologists. Trends Cell Biol 8:397-403.

Li M, Brooks CL, Wu-Baer F, Chen D, Baer R, Gu W (2003) Mono- versus polyubiquitination: differential control of p53 fate by Mdm2. Science 302:1972-1975.

Liu J, Chang L, Roselli F, Almeida OF, Gao X, Wang X, Yew DT, Wu Y (2010) Amyloid- $\beta$ induces caspase-dependent loss of PSD-95 and synaptophysin through NMDA receptors. J Alzheimers Dis 22:541-556.

Lu X, Rong Y, Baudry M (2000) Calpain-mediated degradation of PSD-95 in developing and adult rat brain. Neurosci Lett 286:149-153.

Man HY, Lin JW, Ju WH, Ahmadian G, Liu L, Becker LE, Sheng M, Wang YT (2000) Regulation of AMPA receptor-mediated synaptic transmission by clathrin-dependent receptor internalization. Neuron 25:649-662.

McGee AW, Dakoji SR, Olsen O, Bredt DS, Lim WA, Prehoda KE (2001) Structure of the SH3-guanylate kinase module from PSD-95 suggests a mechanism for regulated assembly of MAGUK scaffolding proteins. Mol Cell 8:1291-1301.

Montgomery JM, Zamorano PL, Garner CC (2004) MAGUKs in synapse assembly and function: an emerging view. Cell Mol Life Sci 61:911-929.

Morabito MA, Sheng M, Tsai LH (2004) Cyclin-dependent kinase 5 phosphorylates the $\mathrm{N}$-terminal domain of the postsynaptic density protein PSD-95 in neurons. J Neurosci 24:865-876.

Mukhopadhyay D, Riezman H (2007) Proteasome-independent functions of ubiquitin in endocytosis and signaling. Science 315:201-205.

Ohshima T, Ogura H, Tomizawa K, Hayashi K, Suzuki H, Saito T, Kamei H, Nishi A, Bibb JA, Hisanaga S, Matsui H, Mikoshiba K (2005) Impairment of hippocampal long-term depression and defective spatial learning and memory in p35 mice. J Neurochem 94:917-925.

Rechsteiner M, Rogers SW (1996) PEST sequences and regulation by proteolysis. Trends Biochem Sci 21:267-271.

Rinehart J, Maksimova YD, Tanis JE, Stone KE, Hodson CA, Zhang J, Ris- inger M, Pan W, Wu D, Colangelo CM, Forbush B, Joiner CH, Gulcicek EE, Gallagher PG, Lifton RP (2009) Sites of regulated phosphorylation that control K-Cl cotransporter activity. Cell 138:525-536.

Schuman EM, Murase S (2003) Cadherins and synaptic plasticity: activitydependent cyclin-dependent kinase 5 regulation of synaptic beta-catenincadherin interactions. Philos Trans R Soc Lond B Biol Sci 358:749-756.

Shumway SD, Maki M, Miyamoto S (1999) The PEST domain of IkappaBalpha is necessary and sufficient for in vitro degradation by mu-calpain. J Biol Chem 274:30874-30881.

Tavares GA, Panepucci EH, Brunger AT (2001) Structural characterization of the intramolecular interaction between the $\mathrm{SH} 3$ and guanylate kinase domains of PSD-95. Mol Cell 8:1313-1325.

Toll H, Oberacher H, Swart R, Huber CG (2005) Separation, detection, and identification of peptides by ion-pair reversed-phase high-performance liquid chromatography-electrospray ionization mass spectrometry at high and low pH. J Chromatogr A 1079:274-286.

Vinade L, Petersen JD, Do K, Dosemeci A, Reese TS (2001) Activation of calpain may alter the postsynaptic density structure and modulate anchoring of NMDA receptors. Synapse 40:302-309.

Wade M, Wang YV, Wahl GM (2010) The 553 orchestra: Mdm2 and Mdmx set the tone. Trends Cell Biol 20:299-309.

Wei FY, Tomizawa K, Ohshima T, Asada A, Saito T, Nguyen C, Bibb JA Ishiguro K, Kulkarni AB, Pant HC, Mikoshiba K, Matsui H, Hisanaga S (2005) Control of cyclin-dependent kinase 5 (Cdk5) activity by glutamatergic regulation of p35 stability. J Neurochem 93:502-512.

Xu W, Schlüter OM, Steiner P, Czervionke BL, Sabatini B, Malenka RC (2008) Molecular dissociation of the role of PSD-95 in regulating synaptic strength and LTD. Neuron 57:248-262.

Zhang S, Edelmann L, Liu J, Crandall JE, Morabito MA (2008) Cdk5 regulates the phosphorylation of tyrosine 1472 NR2B and the surface expression of NMDA receptors. J Neurosci 28:415-424. 